\title{
Neighborhood Effects on Hispanic Young Adult Outcomes*
}

\author{
Benjamin D. Smith \\ Arizona State University, \\ Tempe, USA
}

\author{
Jacquelyn D. Wiersma-Mosley, \\ Lindsay S. Ham, Zola K. Moon \\ University of Arkansas, Fayetteville, USA
}

\begin{abstract}
The present study explored the implications of neighborhood effects and parent-child relationships upon young adulthood attainment among Hispanic adolescents. Examining the social contexts in which Hispanic adolescents develop, such as neighborhoods, allows researchers a greater depth of understanding the processes and potential risks that influence young adulthood attainment. Utilizing The National Longitudinal Study of Adolescent to Adult Health (Add Health), the current study examined neighborhood effects, specifically neighborhood disorganization and perceptions of neighborhood safety, in relation to education and career attainment outcomes in young-adulthood among a representative sample of 1,814 Hispanics. Results of the current study illuminate the mechanisms of neighborhood quality and academic belonging as influencing young adulthood outcome attainment. These findings inform current research and future policy to more effectively support development of Hispanic adolescents.
\end{abstract}

Keywords: Hispanic adolescents, neighborhood disorganization, acculturation, parent-child connectedness

By 2060, the U.S. population will consist of nearly 12.8 million Hispanic persons and will constitute nearly a third of the U.S. population (U.S. Census Bureau, 2012). Examining the social contexts in which Hispanic adolescents develop, such as neighborhoods, allows researchers a greater depth of understanding of the processes and potential risks that influence young adulthood attainment, such as education and career outcomes (Sampson, Morenoff, \& Gannon-Rowley, 2002). The present study incorporates the role acculturation among Hispanic adolescents as they navigate social environments during adolescence. The current study examined neighborhood effects during adolescence as associated with Hispanic young adult outcomes, such as post-secondary education and career attainment. Adolescents' reports of neighborhood cohesion and their parent's perception of neighborhood safety were used to gain an insight into neighborhood disorganization and elements of collective efficacy to combat negative neighborhood elements. These environmental features were assessed in conjunction with additional social influences, such as parent-child relationships, which have been shown to function as a potent role in young adulthood outcome. Parent-child

\footnotetext{
*Acknowledgements: This research was part of the thesis project at the University of Arkansas to the 1st author and was presented at the Society for the Study of Emerging Adulthood in October, 2015. This research uses data from Add Health, a program project directed by Kathleen Mullan Harris and designed by J. Richard Udry, Peter S. Bearman, and Kathleen Mullan Harris at the University of North Carolina at Chapel Hill, and funded by grant P01-HD31921 from the Eunice Kennedy Shriver National Institute of Child Health and Human Development, with cooperative funding from 23 other federal agencies and foundations. Special acknowledgment is due to Ronald R. Rindfuss and Barbara Entwisle for assistance in the original design. Information on how to obtain the Add Health data files is available on the Add Health website (http://www.cpc.unc.edu/addhealth). No direct support was received from grant P01-HD31921 for this analysis.

Benjamin D. Smith, M.S., T. Denny Sanford School of Social and Family Dynamics, Arizona State University.

Jacquelyn D. Wiersma-Mosley, Ph.D., School of Human Environmental Sciences, University of Arkansas.

Lindsay S. Ham, Ph.D., School of Human Environmental Sciences, University of Arkansas.

Zola K. Moon, Ph.D., Department of Psychological Science, University of Arkansas.
} 
relationships are of particular note with regards to cultural aspects relevant to Hispanic youth (i.e., "familismo", Perez-Brena, Updegraff, \& Umana-Taylor, 2014).

Social disorganization theory (see Sampson, Morenoff, \& Gannon-Rowley, 2002; see also Leventhal \& Brooks-Gunn, 2000) suggests that specific characteristics of neighborhoods, such as poverty, ethnic heterogeneity, and residential instability, play an active role in increasing the risk of hazardous outcomes such as violence and crime. In disadvantaged neighborhoods, formal social institutions (i.e., formally organized activities which serve to maintain social order within the neighborhood) that would ordinarily bolster the community and promote healthy, prosocial neighborhood activity are either deficient or altogether absent. Informal structures refer to interactions among neighbors such as the propensity for neighborhood adults to monitor the behavior of adolescents in order to preserve social control (assessed in the present study by perceptions of neighborhood cohesion).

Additionally, the life course perspective (see Elder \& Rockwell, 1979) offers a supplementary framework for conceptualizing social role transitions precipitated by exposure to neighborhood crime. This perspective frames the development of individuals within their cohort, transitioning between social contexts based upon physical age and social development throughout the life course (Bengston \& Allen, 1992). Further, this perspective acknowledges the significance of sociohistorical context within which individuals exist.

As the life course perspective suggests, individual, social, and sociohistoric factors function together to guide individuals from one social context to another. With regard to young adulthood attainment, the present study posited that the environment of the neighborhood played a vital role in the life of developing adolescents. From a theoretical perspective, a neighborhood should be a context within which adolescents can form healthy peer relationships and gain from interactions with adult residents. However, if the neighborhood is disorganized (that is, it is not a safe environment with quality social connections), then adolescents would experience a deficit of support in a context that is crucial for access to social capital. This would inhibit adolescents' ability to reach certain goals necessary to reach markers of young adult attainment (for example, career and education outcomes). It is necessary to examine neighborhood factors, as well as individual factors. Acculturation levels and parent-child relationships are of particular interest when considering Hispanic adolescents.

\section{Acculturation}

Acculturation is the process in which the individual confronts, adapts to, and modifies their identity and affiliation in the face of dominant social and cultural values which vary from their cultural heritage (Lee \& Hahm, 2009; Schwartz et al., 2014). This process involves a transition during which individuals come to identify themselves within their new cultural setting while simultaneously bringing values of their culture of origin. This can be a stressful experience and potentially reduces access to sources of support (Rivera, 2007). It is also a particularly arduous experience for adolescents to navigate, as adolescence is already a time of struggle for identity and belonging.

Migrant experiences present a unique purview for examining the social world of developing adolescents. Hispanic social and cultural values generally differ from those of the United States (US), particularly with regards to familial emphasis, or "familismo" (Ayón, Marsiglia, \& Bernudez-Parsai, 2010). Latin-American culture places an emphasis on collectivism, whereas US culture generally promotes individual independence (Schwartz et al., 2014). In collectivist cultures, the self is perceived as being integrated with the identities of other individuals; however, in the US the "self" is typically thought of as an autonomous, separate entity 
(Gilovich, Keltner, \& Nisbett, 2011). An example of collectivistic culture's familial emphasis is multigenerational cohabitation in which three or four generations will reside within a single household. Multigenerational cohabitation is a means of bolstering support among family members and is a common feature in Latin-American households (Djajic, 2003; Ishizawa, 2004).

The influence of immigration timing has been reflected in educational behaviors. For example, cultural factors due to generation location (i.e., 1st generation, 2nd generation immigrant; 1.5 generation being minors who immigrate with their parents, see Farley \& Alba, 2002) may play a vital role in post-secondary enrollment and persistence. For example, previous research indicates that those adolescents who are more acculturated exhibited greater tendencies both with respect to pursuing and persisting in post-secondary education (Castillo, López-Arenas, \& Saldivar, 2010; see also Flores, Ojeda, Huang, Gee, \& Lee, 2006).

It is important to understand how the acculturation process may be related to the development of Hispanic adolescents' emergence into the cultural landscape and norms of the US. As adolescence is a time during which individuals are exposed exceedingly more to others outside of the household, there may be strain upon the adolescent's relationships within the family. Acculturative transitions, such as adolescents' use of English over Spanish, may enhance the stresses of their changing social world, but may also aid in their mobility within the majority culture (i.e., the US). It is important to recognize the process of Hispanic adolescents' sociocultural transitions at the nexus of adolescent identity formation and cultural identity negotiation.

\section{Parent-Family Connectedness}

Family relationships are identified as the primary source of social support which fosters resilience for adolescents (Ali, Dwyer, Vanner, \& Lopez, 2010). Bronfenbrenner (1979) states that as an individual matures and expands their social world, the family remains the "most stable and enduring base throughout this process" (p. 232). The family unit is a source of social support which provides a multitude of protective factors for individuals, such as increasing self-concept (Mueller \& Haines, 2012). The family unit acts as a foundation by which the individual prepares for engagement with the world. Moreover, the protective factor of the family has been shown to decrease a multitude of health-risk behaviors, such as initiation of violent behavior, depression, and substance abuse (Brookmeyer, Fanti, \& Henrich, 2006; Henrich, Brookmeyer, \& Shahar, 2005; Mueller \& Haines, 2012; Resnick, Harris, \& Shew, 1997).

Once again "familismo" may serve a vital protective function in the lives of developing and acculturating Hispanic youth in the US. However, as is evidenced in previous literature (Gonzales et al., 2008), the role of immigrant generational-status causes shifts in motivation towards certain goals, for example adolescents of later generations experiencing a reduction in likelihood to obtain a post-secondary degree. While family remains an important social support system, the manifestation of "familismo's" influence in the lives of Hispanic youth alters as a result of the acculturation process. For example, if the cultural messages and norms embedded in "familismo" become less important to acculturating Hispanic youth, their behavior may reflect these cultural identity changes by reducing their motivation to seek higher education. This may be particularly true when considering specific Hispanic groups. For example, Mexican-origin Americans and Cuban Americans more closely resemble two-parent, single-earner households, while Hispanics of other ethnic groups (e.g., Dominicans) belong to a matrifocal culture (i.e., female-headed households; Cherlin, 2010). Hispanics of Dominican descent are also more likely than other Hispanic groups to live in households headed by females, similar to African American households. These similar feature (matrifocal cultural influence and female-headed 
households) may also increase the likelihood that adolescent Hispanics of Dominican descent will reside in lower socioeconomic status environments (and therefore more likely to be disorganized neighborhoods) due to living in female-headed households.

It is important to identify how the parent-child connectedness might be associated with the acculturation process. It may be the case that as an adolescent becomes more integrated into the dominant culture, the connection with their family wanes. This is speculated to be particularly true among families in which there exists greater cultural incongruence. That is, if an acculturating Hispanic adolescent is identifying more with the dominant culture while their parents identify more with the traditional values of their culture of origin, this may stress the parent-child relationship (Perez-Brena et al., 2014).

\section{Neighborhoods}

Human development literature contains a rich history of research identifying and examining the contexts in which development takes place. Neighborhoods have been at the center of such contexts. Past research suggests that the neighborhood environment plays an increasingly important role in the lives of children as they grow older (Woolley et al., 2008). As the social world of a developing individual expands, they begin to rely on individuals outside of their household. This transition emphasizes the importance of social capital available within the neighborhood. Features of such neighborhood social capital include sharing responsibilities with others and receiving advice from individuals outside of one's family (Wooley et al., 2008).

Although the literature is rather dense, meta-analyses (see Sampson et al., 2002) indicate that the complexities involved in identifying specific factors of neighborhoods and their implications upon development require further attention. Examining neighborhood effects involves sifting through a variety of neighborhood characteristics and identifying how these characteristics function together to impact development and outcomes later in life. Characteristics of the neighborhood have major implications upon a variety of issues such as overall social capital, parent-child relationships, academic achievement, sexual behaviors, mental health, and more (Bowen, Bowen, \& Ware, 2002; Campos, 2006; Cubin, Santelli, Brindis, \& Braveman, 2005; Vega, Ang, Rodriguez, \& Finch, 2011; Woolley et al., 2008).

\section{Neighborhood Disorganization}

With the increasing number of Hispanic immigrants living in the United States, researchers have begun examining the impact of ethnic concentration and cultural influences upon neighborhood effects. Past research demonstrates that non-Caucasian and low-income families are at greater risk for living in socially disorganized neighborhoods (Bowen et al., 2002). Neighborhood disorganization refers to dissolution of prosocial elements within the neighborhood, such as clubs or other organized activities that promote interaction and social support, and the increased risk of deviant behavior (i.e., substance problems and violence; Bowen Bowen et al., 2002). Bowen et al.'s (2002) research revealed that perceptions of parenting practices are influenced by the level of neighborhood disorganization. This is further evidence that socially disorganized neighborhoods negatively influence parenting styles by hindering parents' ability to tend to the needs of their children. Middle school and high school age children reported perceiving their parents as less supportive and in turn this negatively affected children's perception of education support received from their parents. This provides further support for the notion that neighborhood effects influence academic outcomes by influencing parent-child relationships (Engberg \& Wolniak, 2010). 
Studies that highlight the importance of cultural values suggest ethnic background and the interchange between dominant and minority cultures may have both positive and negative implications. Behavioral expectations imbedded in Hispanic culture may be protective against risky behaviors for adolescents. For example, Hispanic female adolescents living in neighborhoods with a high concentration of Hispanic families report lower levels of initiating sex during adolescence (Cubbin, Santelli, Brindis, \& Braveman, 2005). This outcome is considered to be a result of positive cultural influences such as sharing cultural norms and having access to intergenerational networks of support.

Additional studies illuminate the protective nature of Hispanic cultural influences against mental health issues. Living in high poverty census tracts, which is a common experience among many Hispanic individuals, increases depression levels. Vega et al. (2011) provided evidence that living in a household with high linguistic isolation may reduce depression. "Linguistic isolation" refers to those settings wherein the native language of immigrants is the predominate language spoken. Vega and colleagues (2011) found that depression levels were lower among Hispanics living in high linguistic isolation households in comparison to those living in low linguistic isolation households. This is evidence of cultural homogeny within one's home buffering against environmental disadvantages outside one's household.

Alternatively, living in a high linguistic isolation household was also associated with feeling less close to one's neighbors. The authors posit that as individuals learn English, the social utility of being in a household that predominately speaks a minority language may be reduced. For example, the positive influence of cultural homogeny inside the home may begin to shift downwards as an acculturating adolescent acquires a mastery of the English language. This may suggest that their household environment is then inhibiting the adolescent developing mobility within the majority culture. These findings further explicate the complex experience of the acculturation process. Rather than conceptualizing the acculturation process as an on/off switch experience, the process is a graduated experience of easing into the majority culture. The acculturation process is impacted by social environments and, likewise, experiencing a shift in cultural identity involves a give and take relationship between one's social environments and the support they may offer or inhibit.

Neighborhood safety. Previous research assessing the negative outcomes of neighborhood violence provides grim outcomes of living in dangerous neighborhoods. The detriments of dangerous neighborhoods not only affect individual factors but also affect interpersonal relationships, such as family and peer relationship quality (Harding, 2008; White \& Roosa, 2012). White and Roosa (2012) described the effects of increased levels of perceived neighborhood danger among Mexican-origin fathers. The results of this study indicated that as the perception of neighborhood danger increased, family cohesion decreased. These circumstances served to increase family stress, which resulted in adolescent internalizing symptoms. Other studies supported these findings and identified additional repercussions of other components of neighborhood disorganization, such as perceptions of safety, cohesion, and socioeconomic status (SES). Neighborhood disorganization is related to increased negative school behaviors, such as poor grades and attrition (Bowen et al., 2002). The same research discovered neighborhood disorganization decreased youths' perceptions of their parents as being supportive of their education. Neighborhood disorganization, specifically threats to neighborhood safety, has the potential to cause significant breaches in the parent-child relationship.

Neighborhood cohesion. Neighborhoods and schools have a complex relationship. Neighborhood characteristics strongly predict students' education outcomes on multiple levels. Utilizing Add Health data (Owens, 2010), SES has been found to be one of the strongest neighborhood characteristics for predicting both 
high school graduation and postsecondary enrollment. Specifically, students who live in high SES neighborhoods may have more people in this environment who have experience with postsecondary education. This concept exemplifies the importance of social capital and having access to other people in the immediate environment who are able to transfer useful information to developing individuals. Owens (2010) accentuates the role of the neighborhood as a context which continues to play an influential social role even after previous school peer groups dissolved once an adolescent graduates from secondary school.

In accordance with social disorganization theory, additional studies have shown that living in disadvantaged neighborhoods affects internalizing behaviors. Residents in disadvantaged neighborhoods are less likely to view themselves as being capable of achieving a postsecondary education (Stewart, Stewart, \& Simons, 2007). However, in a study examining neighborhood cohesion among African American adolescents, Stewart et al. (2007) found that neighborhood cohesion increased the likelihood that adolescents would express confident postsecondary aspirations, such as believing that they would attend college. The results of this study illuminated the role of neighborhood cohesion as a protective mechanism despite other elements of neighborhood disorganization, such as a lack of safety.

In a study examining neighborhood influences on parental involvement in adolescents' education, researchers identified Hispanic American parents to be overall less involved than their white peers (Crosnoe, 2001). Neighborhood characteristics, such as maternal employment and education level, proved to be more instrumental than school characteristics for predicting parental education involvement. Hispanic individuals are more likely to live in disorganized neighborhoods and therefore Hispanic adolescents are more likely to experience a deficit in parental education involvement. Moreover, the overall education level of neighborhood residents influences the tendency that neighborhood parents will attend school functions. This suggests a collaborative element of the neighborhood functioning as a collective unit to influence the lives of child residents. This is perhaps similar to when neighborhood children interact with neighborhood adults and use them as resources by relying on adults' experiences (i.e., social capital), such as academic or career attainment, to learn more about possible goals to work towards. This is suggestive of neighborhood environments influencing individual factors, for example parental involvement or interactions with other, as attributes of an overall cohesive feature of the neighborhood.

These results may accentuate the utility of neighborhood social cohesion to protect developing adolescents from certain social disadvantages. Again, these results support the concept that an adolescent's relationship with other individuals in their neighborhood may be crucial for bolstering success. This indicates that neighborhood cohesion is important for protecting neighborhood children against neighborhood disadvantages and may also be important for promoting healthy behaviors which carry on into young adulthood.

The current study aimed to identify how neighborhood cohesion and safety for Hispanic adolescents functioned simultaneously as a substantial assessment of neighborhood characteristics with regards to predicting outcomes in young adulthood, above and beyond the effects of acculturation and parent-child connectedness. As such, the current study addressed gaps in the literature in isolating the role of neighborhood cohesion and safety in education and career development among Hispanic young adults. This studyhypothesized higher neighborhood safety and cohesion would be positively associated with young adult outcomes (specifically, college enrollment and occupation status) for Hispanic individuals. Exploratory analyses also examined potential group differences in neighborhood experiences among Hispanic ethnic groups. 


\section{Method}

\section{Participants}

Data were from the National Longitudinal Study of Adolescent to Adult Health (Add Health; Harris, 2009; see Harris, Halpern, Whitsel, Hussey, Tabor, Entzel, \& Udry, 2009, for design information), which is a school-based, nationally representative and longitudinal study that began in 1994 and assessed health-related behaviors of adolescents and later outcomes. This study used Wave I (1994-1995) that included Hispanic adolescent reports of parent-family connectedness, neighborhood cohesion, and their parents' (mostly mothers) perceptions of neighborhood safety, and also used Wave III (2001-2002) data that included the adolescents' education and career attainment outcomes during young adulthood. Participants included Hispanic adolescents (and their parents), $N=1,814$ (51\% girls), with a mean age of 15.5 at Wave I and 21.95 ( $S D=1.65$; Range 18-27) at Wave III. At Wave I, adolescents identified as Mexican American (51\%), Chicano (5\%), Cuban (14\%), Puerto Rican (17\%), Central/South American (10\%), and other Hispanic-origin (9\%).

\section{Measures}

Acculturation. The measure of acculturation in the Wave I In-Home Interview was the adolescent report of the language spoken at home (Lee \& Hahm, 2009). Language was employed as a measurement of acculturation because it can serve as an indicator of ethnic identity (Kang, 2006). Regarding language, Hispanic adolescents were asked, "What language is usually spoken in your home?" and reported either 1 = "Spanish", 2 $=$ "English". For this sample, 55\% spoke English and 44\% spoke Spanish.

Parent-family connectedness. The In-Home Interview included a six-item parent-family connectedness scale assessed in Wave I (Mueller, 2009). Hispanic adolescents were asked to report their perceptions of parent-family connectedness prompted by statements which included: "How close do you feel to your mother/father", "How much do you feel that your parents care about you", and "How much do you feel that your family pays attention to you" Each item was scored on a 5-point Likert ranging from $0=$ "Not at all" to 5 $=$ "Very much". These items were averaged into a single, internally consistent measure with a mean of 3.15 $(S D=0.66)$. Cronbach's alpha was equal to 0.80 .

Neighborhood safety. The In-Home Interview included a seven-item neighborhood safety scale assessed in Wave I (Benson \& Faas, 2014). Hispanic adolescents' "parents" were asked to report their perceptions of neighborhood safety prompted by several statements with varying Likert scale responses. These statements included questions such as: "You live here because there is less crime in this neighborhood than there is in other neighborhoods" and "You live here because there is less substance use and other illegal activity by adolescents in this neighborhood". These items required "No" $=1$, or "Yes" $=2$ responses. Additional questions such as: "In this neighborhood, how big a problem are drug dealers and substance abuse?" consisted of scaled responses ranging from "No problem at all" or "Not at all" = 3 to "A big problem" or "Very much" = 1. Each item was scored on a Likert scale. Items were reverse-scored and standardized into a single, internally consistent measure with a mean of $0.05(S D=4.28)$. The standardized Cronbach's alpha was equal to 0.74 .

Neighborhood cohesion. Measures of neighborhood cohesion were assessed by combining six items composed of Hispanic adolescent perceptions and attitudes about the neighborhood and neighborhood interactions. These items were collected during the Wave I In-Home Interview. Similar to neighborhood safety measures, item responses were Likert scales that varied in scaling size. Questions, such as: "You know most of the people in your neighborhood" and "People in this neighborhood look out for each other" were answered in 
the form of "True" $=2$ or "False" $=1$ ratings. These items were based upon previously combined-item measurements of neighborhood cohesion (Deutsch, Crockett, Wolff, \& Russell, 2012); however, the current study found that Cronbach's alpha increased by adding two additional items, such as: "On the whole, how happy are you with living in your neighborhood?" and "If, for any reason, you had to move from here to some other neighborhood, how happy or unhappy would you be?" Responses for these two additional measurements ranged from "Very much" or "Very unhappy" $=5$ to "Not at all" or "Very happy" = 1 . All items were standardized with an internal consistent measure with a mean of $-0.32(S D=3.75)$. The standardized Cronbach's alpha was equal to 0.66 .

Education outcome. In Wave III, Hispanic young adults reported the highest grade or year of regular school they had completed. Responses were coded on a 0 to 3 scale: "No high school diploma" $=0$, "Having a high school diploma" $=1$, "Completing between one and five years of college" $=2$, and "Completing one to five or more years of graduate school" $=3$.

Career outcome. In Wave III, Hispanic young adults reported their current status of occupation. Participants were asked "Are you currently working for pay for at least 10 hours a week?" Responses were coded as either $0=$ "No" or $1=$ "Yes".

Control variables. Additional control variables were utilized, which included parents' level of education ( $M=0.87, S D=0.94$, range: 0 indicating no high school attendance to 3 indicating graduate school and beyond), participant gender, age, and participant GPA averaged over Wave I and II ( $M=2.59, S D=0.72$, range: 1 indicating a $D$-average or lower to 4 indicating an $A$-average). All study variables are shown in Table 1 .

Table 1

Study One Means and Standard Deviations $(n=1,814)$

\begin{tabular}{lrlc}
\hline Variable & $M$ & $S D$ & Range \\
\hline Wave III Age & 21.95 & 1.65 & 9 \\
Parental education & 0.87 & 0.94 & 3 \\
GPA & 2.60 & 0.72 & 3 \\
Language & 1.55 & 0.50 & 1 \\
Connectedness & 3.15 & 0.66 & 3.83 \\
Cohesion & -0.32 & 3.75 & 16.60 \\
Safety & 0.05 & 4.28 & 17.20 \\
Education & 1.26 & 0.76 & 3 \\
Employment & 0.78 & 0.42 & 1 \\
\hline
\end{tabular}

\section{Results}

Two hierarchical regression analyses were used to investigate factors influencing education and career outcomes among Hispanic young adults, as shown in Tables 2 and 3. Model one of the regression analysis predicting employment outcomes included control variables (participant age at Wave III, gender, GPA, and parental education). Participant age, gender, and GPA (but not parental education) were found to be significant predictors of employment status, as shown in Table 2. Language was added in the second model and accounted for a significant proportion of the variance in employment status over and above the effects of participant age at Wave III, gender, GPA, and parental education, $b=-0.07, S E=0.02, p=0.01$. Parent-family connectedness was added in the third model and was approaching significance in accounting for a proportion of the variance in employment status over and above the effects of participant age at Wave III, gender, GPA, parental education, 
and language, $b=0.03, S E=0.02, p=0.06$. Neighborhood cohesion and neighborhood safety were added in the final model, however neither variables accounted for a significant proportion of the variance in employment status over and above the other variables. Because there were no significant neighborhood effects, Hispanic group differences were not assessed.

Table 2

Hierarchical Regression Analysis for Variables Predicting Employment Status $(n=1,814)$

\begin{tabular}{|c|c|c|c|c|c|c|c|c|c|c|c|c|}
\hline \multirow[b]{2}{*}{ Variable } & \multirow[b]{2}{*}{$B$} & \multicolumn{2}{|c|}{ Model 1} & \multicolumn{3}{|c|}{ Model 2} & \multicolumn{3}{|c|}{ Model 3} & \multicolumn{3}{|c|}{ Model 4} \\
\hline & & $S E B$ & $\beta$ & $B$ & $S E B$ & $\beta$ & $B$ & $S E B$ & $\beta$ & $B$ & $S E B$ & $\beta$ \\
\hline Age & 0.02 & 0.01 & $0.09^{* * *}$ & 0.02 & 0.01 & $0.08^{* *}$ & 0.02 & 0.01 & 0.08 & 0.02 & 0.01 & $0.08^{* *}$ \\
\hline Gender & -0.04 & 0.02 & $-0.05^{*}$ & -0.04 & 0.02 & $-0.05^{*}$ & -0.04 & 0.02 & -0.05 & -0.04 & 0.02 & -0.05 \\
\hline $\begin{array}{l}\text { Parental } \\
\text { Education }\end{array}$ & -0.01 & 0.01 & -0.02 & 0.00 & 0.01 & -0.005 & 0.00 & 0.01 & 0.01 & -0.00 & 0.01 & -0.00 \\
\hline GPA & 0.06 & 0.02 & $0.11^{* * *}$ & 0.06 & 0.02 & $0.11^{* * *}$ & 0.06 & 0.02 & $0.10^{* * *}$ & 0.06 & 0.02 & $0.10^{* * *}$ \\
\hline Language & & & & -0.07 & 0.02 & $-0.08^{* *}$ & -0.06 & 0.02 & $-0.08^{* *}$ & -0.06 & 0.02 & $-0.07^{* *}$ \\
\hline Connectedness & & & & & & & 0.03 & 0.02 & $0.05 \sim$ & 0.03 & 0.02 & $0.05 \sim$ \\
\hline Cohesion & & & & & & & & & & -0.002 & 0.00 & -0.02 \\
\hline Safety & & & & & & & & & & 0.004 & 0.00 & 0.04 \\
\hline$R^{2}$ & 0.02 & & & 0.03 & & & 0.03 & & & 0.03 & & \\
\hline
\end{tabular}

Notes. This table shows the unstandardized and standardized coefficient for each variable added to the model; ${ }^{*} p<0.05, p<$ $0.01,{ }^{* * *} p<0.001 ; \sim$ indicates approaching significance.

Table 3

Hierarchical Regression Analysis for Variables Predicting Education Status $(n=1,814)$

\begin{tabular}{|c|c|c|c|c|c|c|c|c|c|c|c|c|}
\hline \multirow[b]{2}{*}{ Variable } & \multicolumn{3}{|c|}{ Model 1} & \multicolumn{3}{|c|}{ Model 2} & \multicolumn{3}{|c|}{ Model 3} & \multicolumn{3}{|c|}{ Model 4} \\
\hline & $B$ & $S E B$ & $\beta$ & $B$ & $S E B$ & $\beta$ & $B$ & $S E B$ & $\beta$ & $B$ & $S E B$ & $\beta$ \\
\hline Age & 0.03 & 0.01 & $0.06^{*}$ & 0.02 & 0.01 & $0.05^{*}$ & 0.03 & 0.01 & $0.06^{*}$ & 0.03 & 0.01 & $0.06^{*}$ \\
\hline Gender & 0.09 & 0.04 & $0.06^{* *}$ & 0.09 & 0.04 & $0.06^{* *}$ & 0.10 & 0.04 & $0.07^{* *}$ & 0.10 & 0.04 & $0.07^{* *}$ \\
\hline $\begin{array}{l}\text { Parental } \\
\text { Education }\end{array}$ & 0.17 & 0.02 & $0.21^{* * *}$ & 0.18 & 0.02 & $0.22^{* * *}$ & 0.18 & 0.02 & $0.22^{* * *}$ & 0.18 & 0.02 & $0.22^{* * *}$ \\
\hline GPA & 0.34 & 0.03 & $0.32^{* * *}$ & 0.34 & 0.03 & $0.32^{* * *}$ & 0.33 & 0.03 & $0.32^{* * *}$ & 0.33 & 0.03 & $0.31^{* * *}$ \\
\hline Language & & & & -0.06 & 0.04 & -0.04 & -0.06 & 0.04 & -0.04 & -0.05 & 0.04 & -0.03 \\
\hline Connectedness & & & & & & & 0.05 & 0.03 & 0.04 & 0.05 & 0.03 & 0.04 \\
\hline Cohesion & & & & & & & & & & 0.00 & 0.01 & -0.01 \\
\hline Safety & & & & & & & & & & 0.02 & 0.00 & $0.10^{* * *}$ \\
\hline$R^{2}$ & 0.17 & & & 0.17 & & & 0.17 & & & 0.18 & & \\
\hline
\end{tabular}

Notes. This table shows the unstandardized and standardized coefficient for each variable added to the model; ${ }^{*} p<0.05,{ }^{* * *} p<$ $0.01,{ }^{* * *} p<0.001$.

The second hierarchical regression analysis examined variables predicting education status. Model one of the regression analysis included control variables (participant age at Wave III, gender, GPA, and parental education). Language was added in the second model, however it did not account for a significant proportion of the variance in education status over and above the effects of participant age at Wave III, gender, GPA, and parental education. Parent-family connectedness was added in the third model, however it did not account for a significant proportion of the variance in education status. Neighborhood cohesion and neighborhood safety were added in the final model. Neighborhood cohesion did not account for a significant proportion of the variance in education status over and above the other variables. However, neighborhood safety did account for 
a significant proportion of the variance in education status over and above the effects of participant age at Wave III, gender, GPA, parental education, language, and connectedness, $b=0.02, S E=0.00, p<0.001$.

Regression analyses were run for each Hispanic ethnic group in effort to identify interactions between groups on neighborhood safety by group on the outcome of young adult educational attainment. Each Hispanic ethnic group was compared to each other Hispanic ethnic group (i.e., Mexican-American vs. Puerto Rican-American) using dummy codes, however, no significant differences emerged.

\section{Discussion}

The results of the current study suggested that neighborhood effects influence Hispanic young adulthood attainment in distinct ways. The only significant predictor beyond the control variables was neighborhood safety. This is particularly interesting when considering Bowen and colleagues' (2002) research which indicates that disorganized neighborhoods increase the likelihood of poor academic performance and attrition. In the current sample, parents' perception of neighborhood safety predicted post-secondary education attainment for young adult Hispanics. However, the reverse of this situation (i.e., living in a dangerous neighborhood) would imply detrimental outcomes for adolescents. The absence of neighborhood safety queues an adolescent up for compounded negative effects, such as not finishing high school or attending college. In other words, the risks associated with even "perceiving" that one's neighborhood is not safe can result in low academic performance and attrition in adolescence and therein an increase in the likelihood that an adolescent will be at risk for depression, anxiety, and substance use (Bond et al., 2007).

Neighborhood safety seems to be a particularly salient influence in predicting education outcomes, whereas this was not the case for employment status. Likewise, cohesion within an adolescent's neighborhood did not show evidence of impacting these outcomes within the present analyses. Participant age, gender, and GPA (but not parental education) were found to be significant predictors of employment in young adulthood. These findings indicated that older Hispanic males with higher high school GPA were more likely to be employed. With regards to acculturation, the current findings suggested that language exposure within one's household played an important role for employment outcomes. Living in a household in which Spanish was the primary language spoken was related to achieving employment. From the perspective of acculturation in relation to language-use, it may be the case that less acculturation is synonymous with more adherence to traditional Latino values. This may also explain the positive association between being male and being employed as values embedded in Latino culture, such as "familismo" or even aspects of "machismo", may proliferate masculine achievement, status, or drive to be the family "breadwinner". Further, these findings supported Vega and colleagues' (2011) research that linguistic isolation was a protective factor in general.

The current study utilized English as an assessment of acculturative status (Lee \& Hahm, 2009). Households wherein Spanish was the dominate language spoken may have indicated less acculturated parents. Language and parent-family connectedness were both found to be significant predictors of employment status in this sample. If it is the case that the use of the Spanish language was a sign of lower acculturation status, then it may have been an indicator that language was acting as a protective agent of socialization. Issues of cultural incongruence between children and parents would require more refined measurements of language and acculturation (see Vega et al., 2011), and this was only found when predicting employment status. Again, these results suggested affiliation with Latino values functions as a protective feature rather than an opposing force with regard to young adulthood attainment. 
Parent-family connectedness in adolescence predicted employment outcomes although neighborhood safety and cohesion were not found to be significant. This finding reinforced previous theory and research (Bronfenbrenner, 1979; Brookmeyer, Fanti, \& Henrich, 2006) stressing that a sense of connection and belonging within the family unit played a potent role in adolescent development and young adult outcomes. Future analysis may consider how these variables may be involved in employment trends specific to Hispanic young adults.

For education outcomes, participant age, gender, parental education, and GPA were all significant predictors of education status. Similar to employment status, results indicated that older Hispanic males with higher GPAs and whose parents were more educated were more likely to have better education outcomes.

\section{Strengths, Limitations and Future Directions}

Considerable strengths of the present study included factors of external validity. The Add Health is a nationally representative longitudinal study which provided the present study with a sample of nearly 2,000 Hispanic individuals and an even gender distribution. The use of Add Health data allowed for an examination of long-term effects of neighborhood effects among a representative sample of Hispanic adolescents. Likewise, although no significant group differences emerged, the sample consisted of a diverse, representative conglomeration of Hispanic ethnic groups in the US. Future neighborhood research should make an effort to more precisely identify which mechanisms within the neighborhood possess the strongest impact for influencing perceptions of safety and understanding the risks associated with both subjective and objective neighborhood safety and residual consequences. Further, the current and future findings may aid educators in evaluating at-risk adolescents.

With regards to assessments of outcome variables, the current study benefitted from being able to identify multiple levels of education attainment. With regards to predicting employment outcomes, some effects were quite small and should be understood as such. Small effects from large-scale surveys are a starting point for more targeted investigations. Alternatively, when predicting education outcomes, the present study produced greater effect sizes.

A limitation of the present study was the inability to comprehensively examine the issue of acculturation. The process of confrontation, adaptation, and modification to one's identity and affiliation with social and cultural values disparate from one's cultural heritage (Lee \& Hahm, 2009; Schwartz et al., 2014) cannot be summed in a single measurement of language. Future examinations of acculturation status would benefit from considering multiple elements of this process, such as generational status, timing of immigration, affiliation with Latino cultural values, nativity of adolescent, and parental nativity and language. Additionally, neighborhood safety assessments would benefit from combining more specific measurements of neighborhood mechanisms that increase or decrease perceptions of neighborhood safety. Future neighborhood measures may also consider including both subjective and objective assessments of neighborhood risk (see Harding, 2008; see also White, Roosa, \& Zeiders, 2012).

Future studies would benefit from identifying more aspects of occupational status. One direction which forthcoming research may consider are the types of jobs in which Hispanic young adults are employed. It will be crucial for future researchers to consider the role of immigrant experiences with regard to employment and education outcomes together, as a mere $67 \%$ of Hispanics 16 and older are employed, with nearly a quarter of the Hispanic population living below the poverty threshold and only $62 \%$ of having completed high school or 
its equivalent, $23 \%$ having completed or enrolled in a two-year degree or some college, and a mere $14 \%$ having completed or been enrolled in a Bachelor's degree program (Pew Research Center, 2013).

The present study highlighted the influential role of neighborhoods among a representative sample of Hispanic adolescents living in the U.S. With the influx of this population in the U.S., the opportunity for future research to identify influential aspects of social contexts and inform policy is plentiful. Future studies have immense opportunity to gain insight into the experiences of a growing portion of the U.S.'s population and create opportunity for a young and developing ethnic group.

In conclusion, the current study reiterated the importance of contexts in the world of developing Hispanic adolescents. These results stressed the importance of a sense of belonging and connectedness of an adolescent to their family. Likewise, these results highlighted the influence of perceptions of safety within one's community and how it had the potential to impact later young adult behaviors, such as education.

\section{References}

Ali, M. M., Dwyer, D. S., Vanner, E. A., \& Lopez, A. (2010). Adolescent propensity to engage in health risky behaviors: The role of individual resilience. International Journal of Environmental Research and Public Health, 7, 2161-2176.

Ayón, C., Marsiglia, F. F., \& Bermudez-Parsai, M. (2010). Latino family mental health: Exploring the role of discrimination and familismo. Journal of Community Psychology, 38(6), 742-756.

Bengtson, V. L., \& Allen, K. R. (1993). The life course perspective applied to families over time. In Sourcebook of family theories and methods (pp. 469-504). Springer US.

Benson, M. J., \& Faas, C. (2014). Assessing adolescent contexts: Measuring of families, peers, schools, and neighborhoods. International Journal of Child Adolescent Health, 7(1), 53-69.

Bond, L., Butler, H., Thomas, L., Carlin, J., Glover, S., Bowes, G., \& Patton, G. (2007). Social and school connectedness in early secondary school as predictors of late teenage substance use, mental health, and academic outcomes. Journal of Adolescent Health, 40, 357.e9-357.e18.

Bowen, N. K., Bowen, G. L., \& Ware, W. B. (2002). Neighborhood social disorganization, families, and the educational behavior of adolescents. Journal of Adolescent Research, 17(5), 468-490.

Bronfenbrenner, U. (1979). The ecology of human development: Experiments by nature and design. Cambridge, MA: Harvard University Press.

Brookmeyer, K. A., Fanti, K. A., \& Henrich, C. C. (2006). Schools, parents, and youth violence: A multilevel, ecological analysis. Journal of Clinical Child and Adolescent Psychology, 25(4), 504-514.

Campos, C. M. T. (2006). Predicting Latino youth academic success from a normative cultural-ecological perspective (Doctoral dissertation). Retrieved from ProQuest Information and Learning Company.

Castillo, L. G., López-Arenas, A., \& Saldivar, I. M. (2010). The influence of acculturation and enculturation on Mexican American high school students' decision to apply to college. Journal of Multicultural Counseling and Development, 38, $88-98$.

Cherlin, A. J. (2010). Demographic trends in the United States: A review of research in the 2000s. Journal of Marriage and Family, 72, 403-419.

Crosnoe, R. (2001). Parental involvement in education: The influence of school and neighborhood. Sociological Focus, 34(4), 417-434.

Cubbin, C., Santelli, J., Brindis, C. D., \& Braveman, P. (2005). Neighborhood context and sexual behaviors among adolescents: Finding from the National Longitudinal Study of Adolescent Health. Perspectives on Sexual and Reproductive Health, 37(3), 125-134.

Deutsch, A. R., Crockett, L. J., Wolff, J. M., \& Russell, S. T. (2012). Parent and peer pathways to adolescent delinquency: Variations by ethnicity and neighborhood context. Journal of Youth and Adolescence, 41, 1078-1094.

Djajić, S. (2003). Assimilation of immigrants: Implications for human capital accumulation of the second generation. Journal of Population Economics, 16, 831-845.

Elder, G. H., \& Rockwell, R. C. (1979). The life-course and human development: An ecological perspective. International Journal of Behavioral Development, 2, 1-21. 
Engberg, M. E., \& Wolniak, G. C. (2010). Examining the effects of high school contexts on postsecondary enrollment. Research in Higher Education, 51(2), 132-153.

Farley, R., \& Alba, R. (2002). The new second generation in the United States. The International Review, 36(3), 669-701.

Flores, L. Y., Ojeda, L., Huang, Y. P., Gee, D., \& Lee, S. (2006). The relation of acculturation, problem-solving appraisal, and career decision-making self-efficacy to Mexican American high school students' educational goals. Journal of Counseling Psychology, 53, 260-266.

Gilovich, T., Keltner, D., \& Nisbett, R. (2011). Social psychology (2nd ed.). S. Snavely (Ed.). New York, NY: W. W. Norton \& Company, Inc.

Gonzales, N. A., Germán, Kim, S. Y., George, P., Fabrett, F. C., Millsap, R., \& Dumka, L. E. (2008). Mexican American adolescents' cultural orientation, externalizing behavior and academic engagement: The role of traditional cultural value. American Journal of Community Psychology, 41(1-2), 151-164.

Harding, D. J. (2008). Neighborhood violence and adolescent friendships. International Journal of Conflict and Violence, 2(1), 28-55.

Harris, K. M. (2009). The National Longitudinal Study of Adolescent to Adult Health (Add Health), Waves I \& II, 1994-1996; Wave III, 2001-2002; Wave IV, 2007-2009 (machine-readable data file and documentation). Chapel Hill, NC: Carolina Population Center, University of North Carolina at Chapel Hill. DOI: 10.3886/ICPSR27021.v9

Harris, K. M., Halpern, C. T., Whitsel, E., Hussey, J., Tabor, J., Entzel, P., \& Udry, J. R. (2009). The National Longitudinal Study of Adolescent to Adult Health: Research Design (WWW document). Retrieved from http://www.cpc.unc.edu/projects/ addhealth/design.

Henrich, C. C., Brookmeyer, K. A., \& Shanhar, G. (2005). Weapon violence in adolescence: Parent and school connectedness as protective factors. Journal of Adolescent Health, 37, 306-312.

Ishizawa, H. (2004). Minority language use among grandchildren in multigenerational households. Sociological Perspectives, $47(4), 465-483$.

Kang, S. (2006). Measurement of acculturation, scale, formats, and language competence: Their implications for adjustment. Journal of Cross-Cultural Psychology, 37(6), 669-693. DOI: 10.1177/0022022106292077.

Lee, J., \& Hahm, H. C. (2009). Acculturation and sexual risk behaviors among Latina adolescents transitioning to young adulthood. Journal of Youth Adolescence, 39, 414-427.

Leventhal, T., \& Brooks-Gunn, J. (2000). The neighborhoods they live in: The effects of neighborhood residence on child and adolescent outcomes. Psychological Bulletin, 126(2), 309-337.

Mueller, C. E. (2009). Protective factors as barriers to depression in gifted and nongifted adolescents. National Association for Gifted Children, 53(1), 3-14.

Mueller, C., \& Haines, R. T. (2012). Adolescent perceptions of family connectedness and school belonging: Links with self-concept and depressive symptoms among gifted African American and Hispanic youth. Gifted Children, 5(2).

Owens, A. (2010). Neighborhoods and schools as competing and reinforcing contexts for educational attainment. American Sociological Association, 83(4), 287-311.

Perez-Brena, N. J., Updegraff, K. A., \& Umana-Taylor, A. J. (2014). Youths' imitation and de-identification from parents: A process associated with parent-youth cultural incongruence in Mexican-American families. Journal of Youth and Adolescence, 43, 2028-2040. DOI: 10.1007/x10964-014-0101-3.

Pew Research Center: Hispanic trends. (2013). Statistical Portrait of Hispanics in the United States, 1980-2013. Retrieved from http://www.pewhispanic.org/2015/05/12/statistical-portrait-of-hispanics-in-the-united-states-1980-2013/\#current-education

Resnick, M. D., Harris, K. M., \& Shew, M. (1997). Protecting adolescents from harm: Findings from The National Longitudinal Study of Adolescent Health. The Journal of the American Medical Association, 278(10), 823-832.

Rivera, F. I. (2007). Contextualizing the experience of young Latino adults: Acculturation, social support and depression. Journal of Immigrant Minority Health, 9, 237-244. DOI: 10.1007/s10903-006-9034-6.

Sampson, R. J., Morenoff, J. D., \& Gannon-Rowley, T. (2002). Assessing “neighborhood effects": Social processes and new directions in research. Annual Review of Sociology, 28, 443-478.

Schwartz, S. J., Zamboanga, B. L., Tomaso, C. C., Kondo, K. K., Unger, J. B., Weisskirch, R. S., Ham, L. S., Meca, A., Cano, M. A., Witbourne, S. K., Brittian, A. S., Des Rosiers, S. E., Hurley, E. A., Vazsonyi, A., T., \& Ravert, R. D. (2014). Association of acculturation with drinking games among Hispanic college students. The American Journal of Drug and Alcohol Abuse, 40(5), 359-366.

Stewart, E. B., Stewart, E. A., \& Simons, R. L. (2007). The effect of neighborhood context on the college aspirations of African American adolescents. American Educational Research Journal, 44(4), 896-919. DOI: 10.3102/0002831207308637. 
Vega, W. A., Ang, A., Rodriguez, M. A., \& Finch, B. K. (2011). Neighborhood protective effects on depression in Latinos. American Journal of Community Psychology, 47, 114-126.

White, R. M. B., \& Roosa, M. W. (2012). Neighborhood contexts, fathers, and Mexican American young adolescents' internalizing symptoms. Journal of Marriage and Family, 74(1), 152-166. DOI: 10.1111/j.1741-3737.2011.00878.x

White, R. M. B., Roosa, M. W., \& Zeiders, K. H. (2012). Neighborhood and family intersections: Prospective implications for Mexican American adolescents' mental health. Journal of Family Psychology, 26(5), 793-804. DOI: 10.1037/a0029426.

Woolley, M. E., Grogan-Kaylor, A., Gilster, M. E., Karb, R. A., Gant, L. M., Reischl, T. M., \& Alaimo, K. (2008). Neighborhood social capital, poor physical conditions, and school achievement. Children \& Schools, 30(3), 133-145. 